\title{
Analysis of probe fitting stimulus properties on transient evoked otoacoustic emissions
}

\author{
Authors \\ (PhD), Annelies Bockstael ${ }^{3,4}$ (PhD) \\ ${ }^{1}$ Department of Rehabilitation Sciences, Ghent University, Belgium \\ 2 Department of Ear, Nose and Throat, Ghent University Hospital, Belgium \\ ${ }^{3}$ Department of Information Technology, Ghent University, Belgium \\ ${ }^{4}$ Ecole d'orthophonie et d'audiologie, Université de Montréal, Canada
}

Hannah Keppler ${ }^{1,2}(\mathrm{PhD})$, Ellen Lampo, Sofie Degeest ${ }^{1}(\mathrm{PhD})$, Dick Botteldooren ${ }^{3}(\mathrm{PhD})$, Bart Vinck ${ }^{1}$

\section{Corresponding author}

Hannah Keppler

Ghent University (Hospital)

C. Heymanslaan 10

9000 Ghent

Belgium

Telephone number: 0032/9/33204 08

\section{Background/ aims}

Probe fitting during evoked otoacoustic emission (EOAE) measurements determines the characteristics of the delivered stimulus and measured responses, as well as the noise reaching the probe's microphone. An optimal probe fitting should be aimed for when measuring EOAEs, and similar probe fittings are needed between EOAE measurements within the same subject. The objectives of the current study are first, to evaluate the subjective evaluation of probe fitting stimulus properties, and second, to analyze the effects of different probe fittings on transient evoked otoacoustic emission (TEOAE) response and noise amplitudes in subjects.

\section{Methods}

Regarding the evaluation of probe fitting stimulus properties, the $Q$ methodology was used whereby 18 participants sorted 48 probe fittings differing in stimulus properties in 7 categories from totally unacceptable to certainly acceptable. Further, TEOAE response and noise amplitudes were measured in 34 ears in one baseline condition with optimal probe fitting and eight experimental conditions whereby probe tip size, probe insertion depth, probe positioning and cable positioning were adjusted to create less-than-optimal probe fittings.

\section{Results}

First, it was found that only $19.61 \%$ of all distributions of the probe fittings by the participants had a mutual correlation of at least $70.00 \%$. Almost $60 \%$ of the variance of distributions was explained by 83.33\% of the participants, although most probe fittings significantly differed from other fittings based on spectral broadness. Second, significant differences in TEOAE response and especially noise amplitudes between conditions were found. Further, TEOAE response and noise amplitudes between the baseline and experimental conditions were significantly different depending on ringing and spectral flatness of the stimulus.

\section{Conclusions}

A substantial amount of subjectivity during TEOAE measurements is involved with regard to the evaluation of probe fittings and used stimulus properties to assess the probe fitting. TEOAE response 
but especially noise amplitudes are influenced by varying stimulus properties which stresses the importance of inspecting these parameters prior to or during EOAE measurements.

\section{Highlights}

- A substantial amount of subjectivity is involved in the evaluation of probe fittings of TEOAE measurements.

- There were significant differences in TEOAE response and noise amplitudes between the baseline optimal probe fitting and experimental less-than-optimal probe fitting conditions.

- The accuracy of TEOAE measurements might be hampered by the probe fitting.

\section{Key words}

- Transient evoked otoacoustic emission

- Probe fitting

- $\quad$ Stimulus properties

\section{Oral presentation}

